\title{
Natal and Neonatal Teeth: An Overview of the Literature
}

\author{
Shubhangi Mhaske, ${ }^{1}$ Monal B. Yuwanati, ${ }^{1}$ Ashok Mhaske, ${ }^{2}$ Raju Ragavendra, ${ }^{1}$ \\ Kavitha Kamath, ${ }^{1}$ and Swati Saawarn ${ }^{1}$ \\ ${ }^{1}$ Department of Oral Pathology and Microbiology, Peoples Dental Academy, Bhopal 462037, Madhya Pradesh, India \\ ${ }^{2}$ Department of Surgery, PCMS \& RC, Bhopal 462037, Madhya Pradesh, India
}

Correspondence should be addressed to Monal B. Yuwanati; monal9817@gmail.com

Received 11 May 2013; Accepted 24 June 2013

Academic Editors: R. G. Faix, S. K. Patole, R. J. Schultz, K. Tokiwa, and D. D. Trevisanuto

Copyright ( 2013 Shubhangi Mhaske et al. This is an open access article distributed under the Creative Commons Attribution License, which permits unrestricted use, distribution, and reproduction in any medium, provided the original work is properly cited.

\begin{abstract}
The occurrence of natal and neonatal teeth is an uncommon anomaly, which for centuries has been associated with diverse superstitions among different ethnic groups. Natal teeth are more frequent than neonatal teeth, with the ratio being approximately $3: 1$. It must be considered that natal and neonatal teeth are conditions of fundamental importance not only for a dental surgeon but also for a paediatrician since their presence may lead to numerous complications. Early detection and treatment of these teeth are recommended because they may induce deformity or mutilation of tongue, dehydration, inadequate nutrients intake by the infant, and growth retardation, the pattern and time of eruption of teeth and its morphology. This paper presents a concise review of the literature about neonatal teeth.
\end{abstract}

\section{Introduction}

Natal teeth are teeth present at birth, and "neonatal teeth" are teeth erupted within the first month of life. Premature eruption of a tooth at the time of birth or too early is combined with many misconceptions. They are further accompanied by various difficulties, such as pain on suckling and refusal to feed, faced by the mother and the child due to the natal tooth/teeth. Some families are so superstitious that the afflicted child may be deprived of parental love. The family hopes that the offending teeth be removed as soon as possible.

Natal and neonatal teeth have been a subject of curiosity and study since the time it was first documented by Titus Livius, in 59 BC. Gaius Plinius Secundus (the Elder), in 23 BC, believed that a splendid future awaited male infants with natal teeth. In some countries, the child is considered to be monstrous and bearer of misfortune for example. As per Chinese tradition it is considered as a bad omen for girls [1].

\section{Terminology and Synonyms}

Dentitia praecox, dens connatalis, congenital teeth, fetal teeth, infancy teeth, predeciduous teeth, and precocious dentition are some of the terminologies used previously $[1,9,12$, $21,65]$. Lack of specificity and accuracy in description of the condition leads to subsequent discontinuity of these terms. The analogous terms of "natal" and "neonatal" teeth described by Massler and Savara are now most accepted [4]. These terms broadly describe the teeth that are erupted at birth or shortly thereafter. Although these terms only define the time of eruption and give no hint whether the tooth is a component of primary dentition or whether it is supernumerary, newer synonyms should be explored.

\section{Proposed Classifications}

The natal and neonatal teeth that do not confirm the criteria described for them and erupt within one to three and a half months are called early infancy teeth [66]. Few authors have tried to resolve the controversies in such cases. Spouge and Feasby [66] in 1966 classified, the natal \& neonatal tooth on the basis of developmental stages whereas, Hebling et al. in 1997 classified according to the appearance of each natal tooth into the oral cavity $[67,68]$ (Table 1$)$.

\section{Incidence and Prevalence}

Natal teeth are three times more common than neonatal teeth. The incidence of natal and neonatal teeth ranges 
TABLE 1: Prevalence of neonatal and natal teeth in different populations and studies.

\begin{tabular}{lcc}
\hline Authors & Prevalence & $\begin{array}{c}\text { Number of } \\
\text { children in the } \\
\text { sample }\end{array}$ \\
\hline Magitot, 1876 [2] & $1: 6000$ & 17,578 \\
Puech, 1876 & $1: 30000$ & 60,000 \\
Ballantyne, 1896 [3] & $1: 6000$ & 17,578 \\
Massler and Savara, 1950 [4] & $1: 2000$ & 6,000 \\
Allwright, 1958 [5] & $1: 3408$ & 6,817 \\
Bodenhoff, 1959 [6] & $1: 3000$ & - \\
Wong, 1962 [7] & $1: 3000$ & - \\
Bodenhoff and Gorlin, 1963 [8] & $1: 3000$ & - \\
Mayhall, 1967 [9] & $1: 1125$ & 90 \\
Chow, 1980 [10] & $1: 2000$ to 3500 & - \\
Anderson, 1982 [11] & $1: 800$ & - \\
Kates et al., 1984 [12] & $1: 3667$ & 7,155 \\
Leung, 1986 [13] & $1: 3392$ & 50,892 \\
Bedi and Yan, 1990 [14] & $1: 1442$ & - \\
Rusmah, 1991 [15] & $1: 2325$ & 9,600 \\
To, 1991 [16] & $1: 1118$ & 53,678 \\
De Almeida and Gomide, & $1: 21.6$ & 1,019 \\
1996 [17] & & 34,457 \\
Alaluusua et al., ${ }^{*}$ 2002 [18] & $1: 1000$ & $(1997-2000)$ \\
El Khatib et al., 2005 [19] & $1: 3400$ & 17000 (1984 and \\
\hline
\end{tabular}

${ }^{*}$ Exposed to toxin Finnish population-correlation with exposure to toxin and prevalence of neonatal teeth and natal teeth.

from $1: 2,000$ to $1: 3,500[19,23]$ (Table 2). The radiographic examination is essential to differentiate the premature eruption of a primary deciduous tooth from a supernumerary tooth [69]. Only $1 \%$ to $10 \%$ of natal and neonatal teeth are supernumerary. More than $90 \%$ of natal and neonatal teeth are prematurely erupted deciduous series of teeth, whereas less than $10 \%$ are supernumerary $[17,70,71]$. The supernumerary teeth should always be extracted, but the decision to extract a normal mature natal tooth should be done by taking into account local or general complications and parental opinion.

The most commonly occurs in the mandibular region of central incisors, followed by maxillary incisors, mandibular cuspids or molars, and maxillary cuspids or molars in descending order $[23,72]$ (Table 3). Natal or neonatal cuspids are extremely rare.

There was no difference in prevalence between males and females. However, a predilection for female was cited by some authors. Anegundi et al. reported a $66 \%$ proportion for females against a $31 \%$ proportion for males [47].

\section{Multifactorial Etiology}

Exact etiology for the premature eruption or for appearance of natal and neonatal teeth is not known. In the past, neonatal teeth were merely considered cysts of the dental lamina of the newborn [67]. Normally they appear corniform, white in colour, composed of compact keratin, and projected above the alveolar ridge [73].

It was also suggested that they occur due to inheritance as dominant autosomal trait. Endocrine disturbance resulting from pituitary, thyroid, and gonads also may be one of the key factors. Another hypothesis suggested is that excessive or increased resorption of overlying bone results in early eruption of the natal or neonatal teeth. Poor maternal health, endocrine disturbances, febrile episodes during pregnancy, and congenital syphilis are some of the contributing predisposing factors for the occurrence of natal and neonatal teeth suggested in the literature. However, according to Štamfelj et al. the occurrence of natal teeth associated with agenesis of their primary successors appears to be related to an accelerated or premature pattern of dental development rather than to superficial positioning of the tooth germs [74].

\section{Environmental Predisposing Factors}

Environmental factors could play an important role in eruption of neonatal teeth. Polychlorinated biphenyls (PCBs), polychlorinated dibenzo- $p$-dioxins (PCDDs), and dibenzofurans (PCDFs) seem to cause the eruption of natal teeth [74]. The only environmental factor that may be regarded as a causative factor of natal teeth is the toxic polyhalogenated aromatic hydrocarbons: PCBs, PCDDs, and PCDFs. They are among the most widespread environmental pollutants. They cross the placenta, and concentrations of PCDD/Fs in the adipose tissue of a newborn are correlated with those in mother's milk. The children with natal or neonatal teeth usually show other associated symptoms [38].

\section{Syndromes Associated}

Few syndromes are reported to be associated with natal teeth and neonatal teeth [8]. These syndromes include Ellis-Van Creveld (Chondroectodermal Dysplasia) [75], Pachyonychia Congenital (Jadassohn-Lewandowsky), Hallermann-Streiff (Oculomandibulodyscephaly with Hypotrichosis) [76], Rubinstein-Taybi, Steatocystoma Multiplex, Pierre-Robin, Cyclopia, Pallister-Hall, Short Rib-Polydactyly (type II), Wiedemann-Rautenstrauch (Neonatal Progeria), Cleft Lip and Palate, Pfeiffer, Ectodermal Dysplasia, Craniofacial Dysostosis, Multiple Steatocystoma, Sotos, Adrenogenital, Epidermolysis-Bullosa Simplex including Van der Woude, Down's Syndrome [77], and Walker-Warburg Syndromes [78].

\section{Clinical Presentation}

The natal teeth or neonatal teeth manifest usually with variable shape and size ranging from small, conical and may also resemble normal teeth. The appearance of these teeth is dependent on the degree of maturity, but most of the time they are loose, small, discoloured, and hypoplastic as in the cases presented here. They may show enamel 


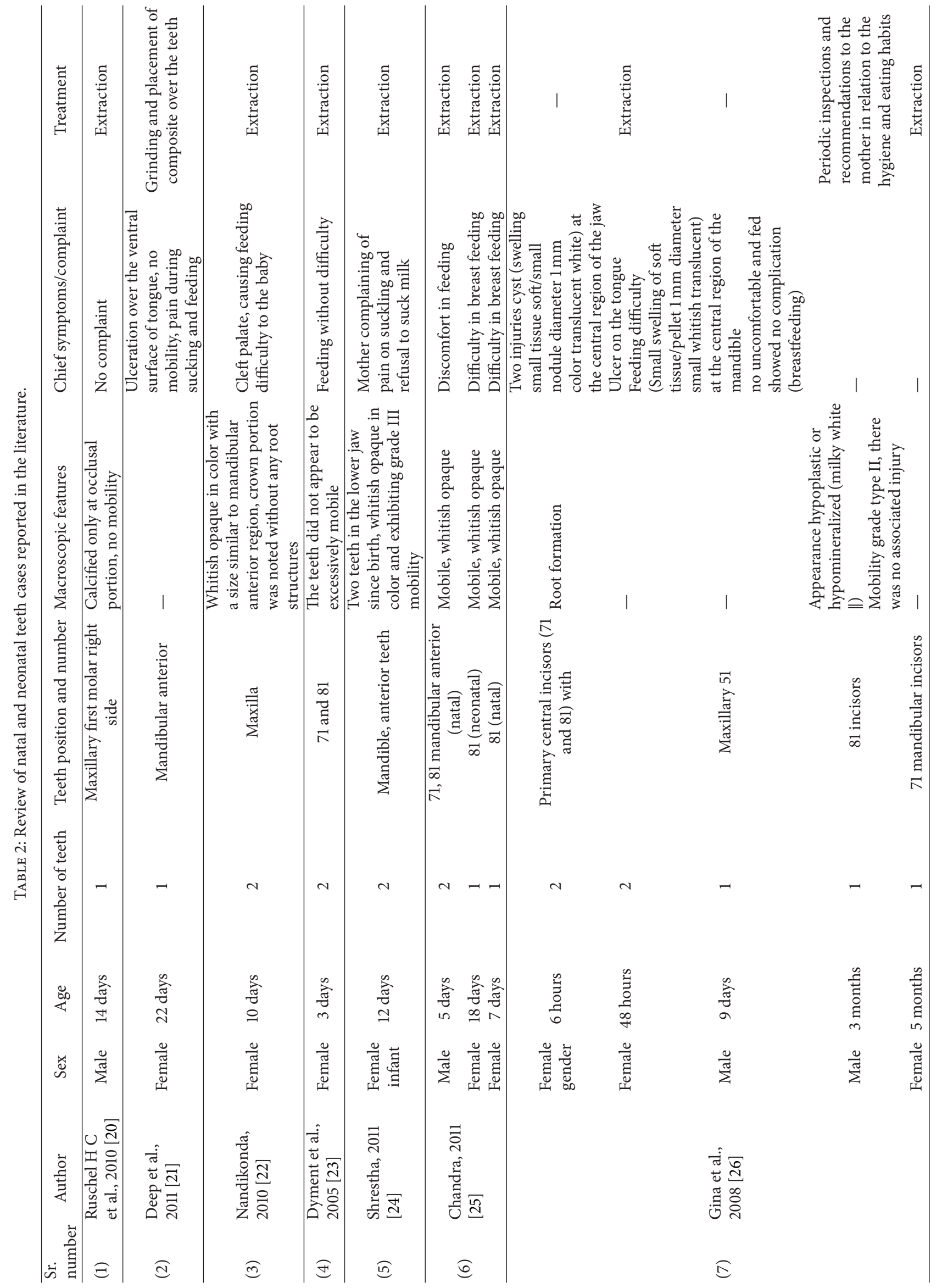




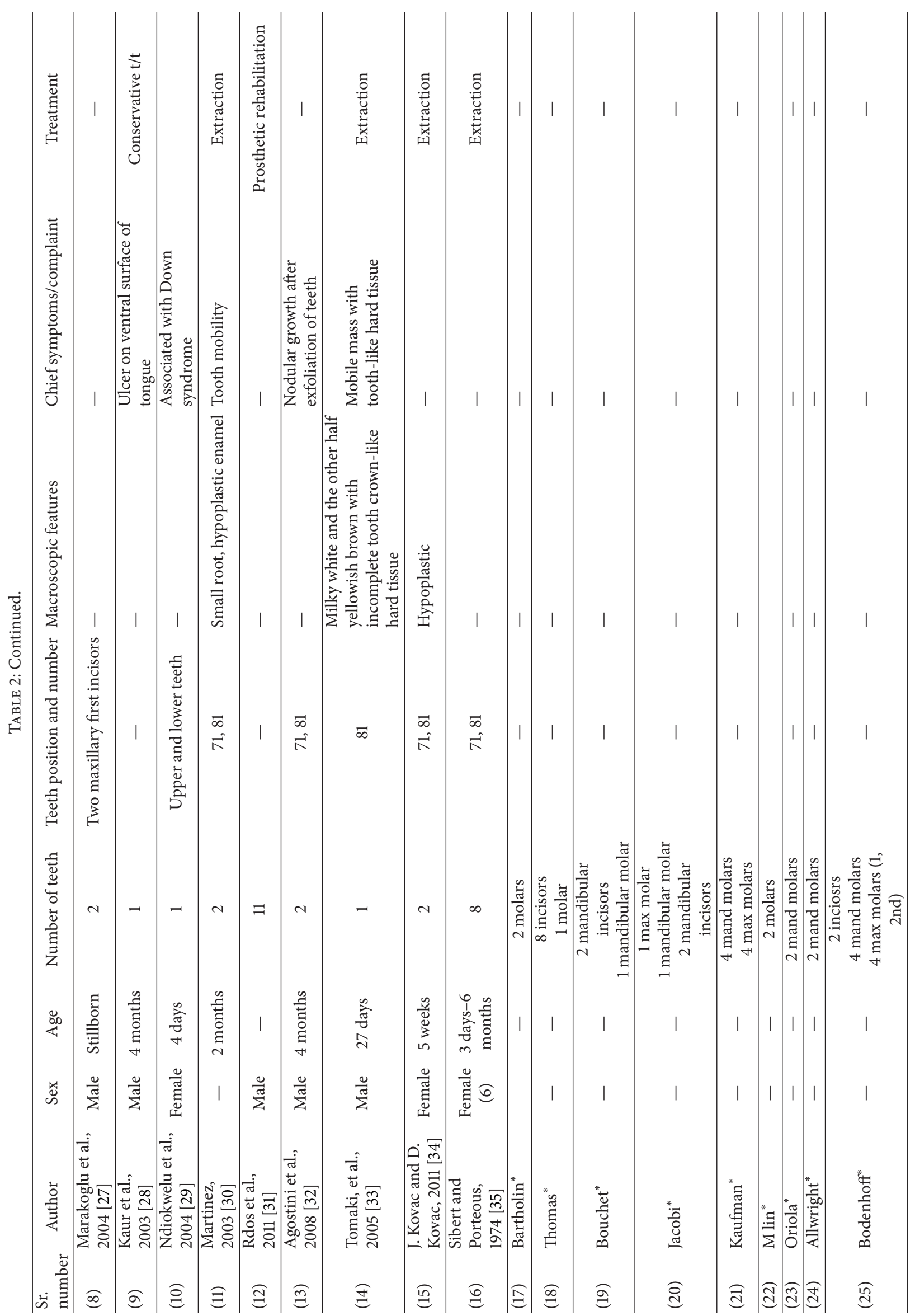




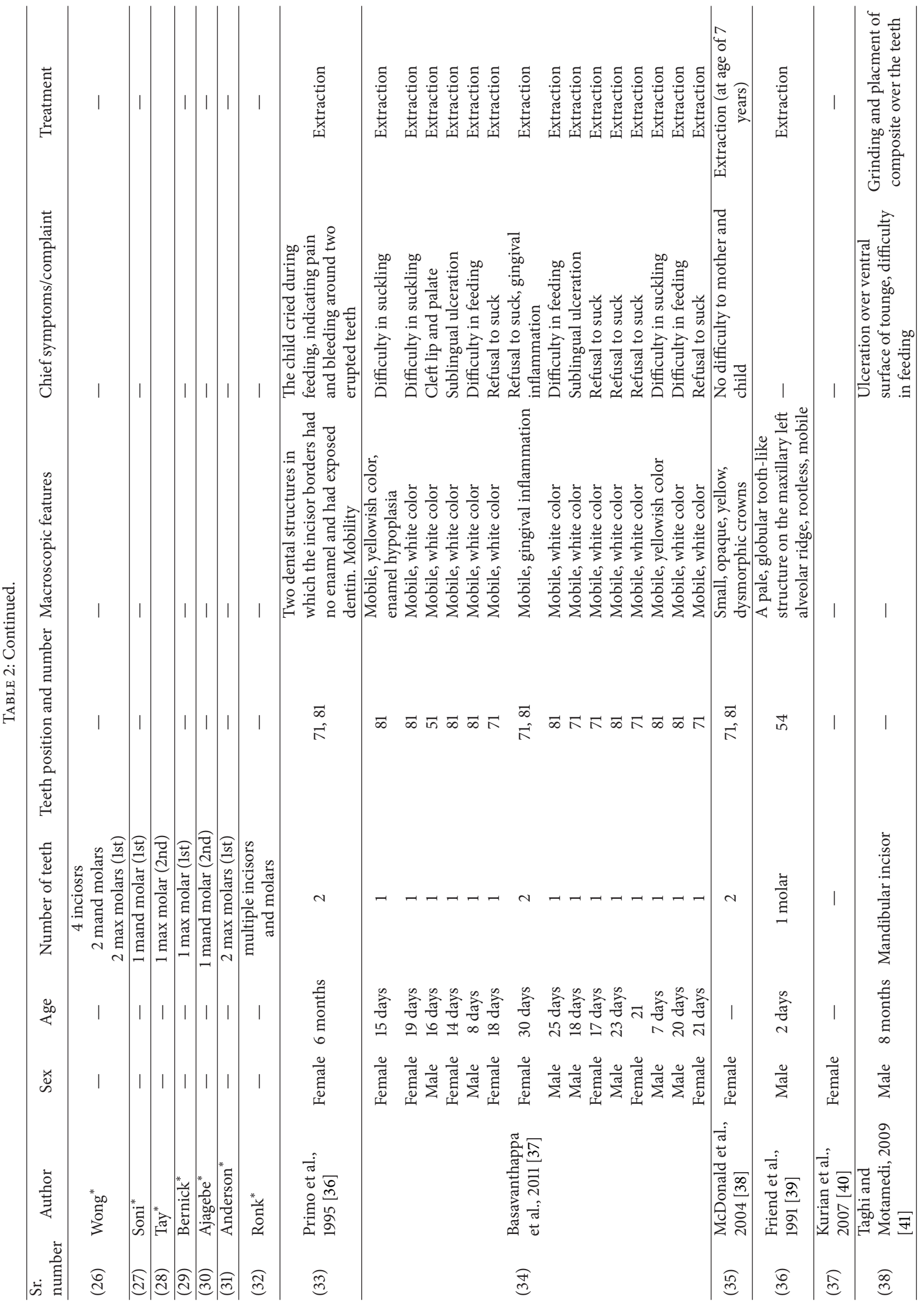




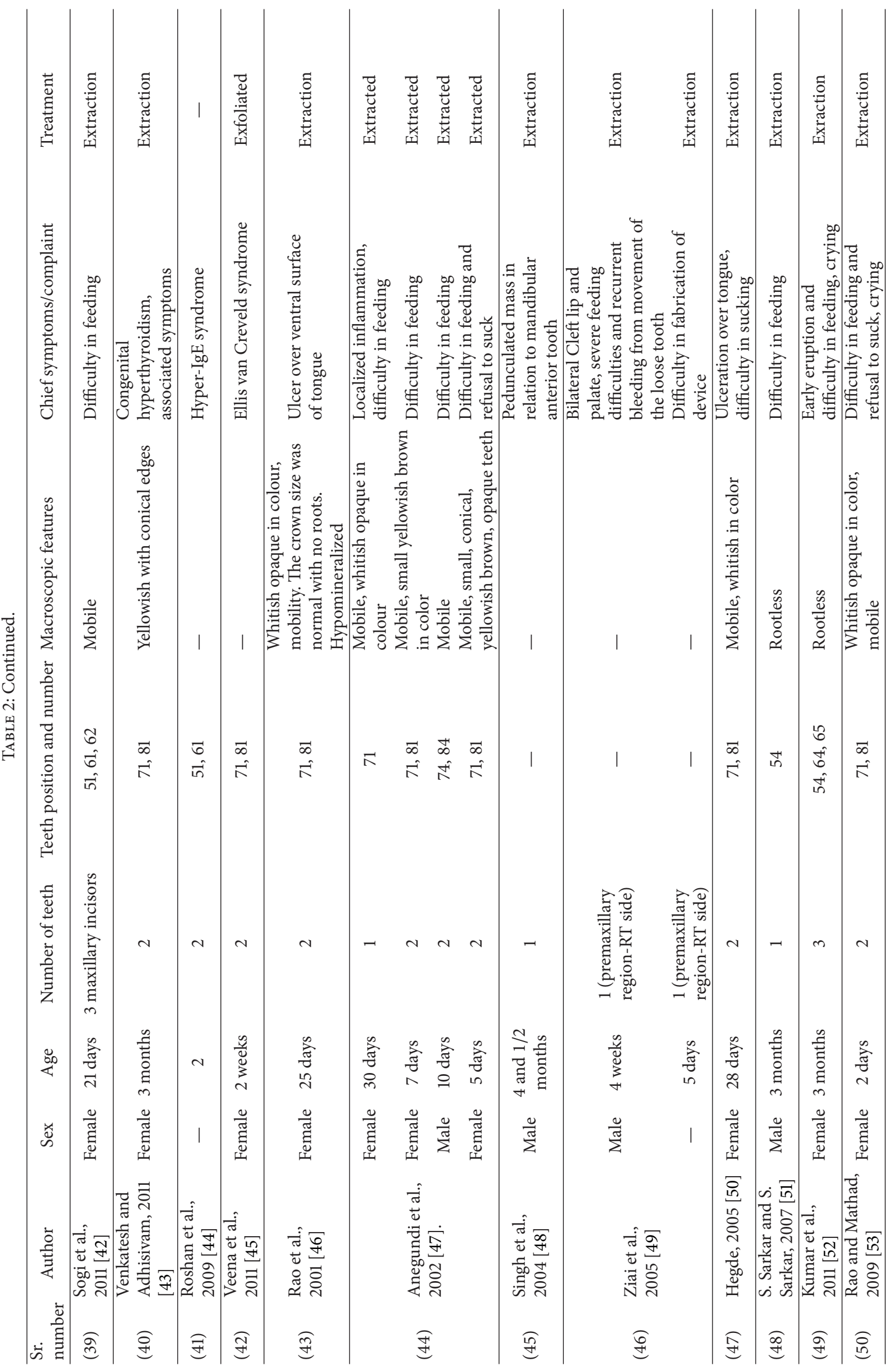




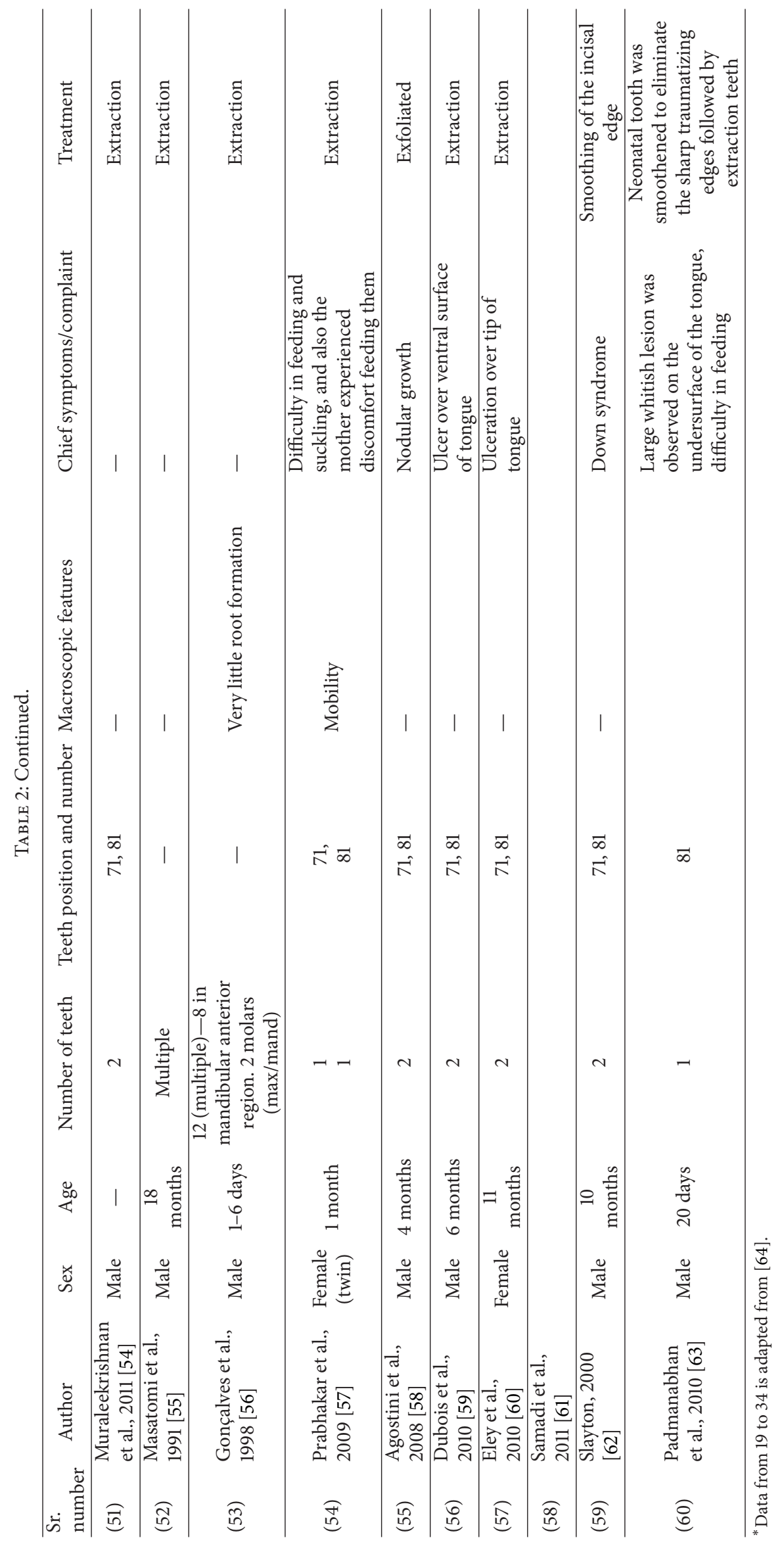


TABLE 3: Details of our cases (total teeth).

\begin{tabular}{|c|c|c|c|c|c|c|}
\hline $\begin{array}{l}\text { Case } \\
\text { number }\end{array}$ & Sex & Age & Teeth position and number & Macroscopic features & Chief symptoms/complaint & Treatment \\
\hline 1 & Male & 5 months & 2 teet & $\begin{array}{c}\text { Yellowish white. Partially formed root. } \\
\text { Size as compared to normal deciduous } \\
\text { central incisor, foramina }\end{array}$ & $\begin{array}{l}\text { Neither the child nor the } \\
\text { mother had any problem } \\
\text { during breast feeding }\end{array}$ & Ext \\
\hline 2 & Male & 3 days & 2 teeth (71 and 81$)$ (natal) & Yellowish white, smaller in size & Difficulty in feeding & Extraction \\
\hline 3 & Male & 2 months & 2 teeth (71 and 81$)$ (neonatal) & $\begin{array}{l}\text { Yellowish white, open apical foramina, } \\
\text { smaller in size }\end{array}$ & $\begin{array}{l}\text { Pain and difficulty in } \\
\text { feeding }\end{array}$ & Extraction \\
\hline
\end{tabular}

hypoplasia/hypomineralization [79] and a small root formation suggestive of an immature nature. The majority of natal teeth may exhibit a brown-yellowish-/whitish-opaque colour [12].

They are attached to the oral mucosa in many instances as the root development is incomplete or defective. This leads to the mobility in teeth, with the risk of being swallowed or aspirated by the child. The mobility also may lead to degeneration of Hertwig's sheath which is responsible for the formation of root, thus resulting in further incomplete root development and stabilization.

Increase in mobility could also cause changes in the radicular part of teeth such as cervical dentin, pulp cavity, and cementum as well.

\section{Histology}

In a study of natal teeth, Hals [80] observed normal pulp tissue, except for the presence of inflammatory areas in some regions; moreover, Weil's basal layer and the cell-rich zone were absent [81]. Histologically, the thin layer of enamel or in extremely rare conditions absence of the enamel layer may be seen [77]. The enamel hypoplasia could be attributed to the disturbance/variation in amelogenesis process which was due to premature exposure of the tooth to the oral cavity. This may cause metaplastic alteration of the epithelium of the normally columnar enamel to a stratified squamous [80].

Dentino-enamal junction is not scalloped which similar to that found in deciduous teeth. Cervically dentin becomes atubular with spaces and enclosed cells [82]. Irregular dentinal tubules through the dentin along with calcospherites and predentin of various thicknesses could be present [33]. Atypical dentin was also observed in the natal/neonatal teeth which could have been the result due to the response to irritant stimulus from oral cavity.

Developing teeth often had no cementum, and in those cases where acellular cementum could be observed it was thinner than normal.

Pulp canal and pulp chamber become wider in most of the cases. Vascularised pulps along with few inflammatory cells were also reported [83].

\section{Ultrastructure Findings}

Jasmin and Clergeau-Guerithault [81] studied the surface topography of mandibular natal and neonatal incisors at the ultrastructural level using the scanning electron microscope
(SEM). They observed that enamel of the teeth exhibited hypoplastic, depressed areas, and the incisal edge of natal tooth lacked enamel [81]. According to Uzamis et al., the thickness of enamel was around 280 microns compared to up to 1200 microns in normal teeth. This shows the retarded development of natal and neonatal teeth, because of incomplete mineralization at the time of birth [82].

In one of such extensive studies on natal and neonatal teeth, Masatomi et al. [55] reported that enamel has a normal prism structure and mineralization except in few cases where the prism structure was absent in the cervical part of the enamel. They also noticed that the cervical and apical dentin was tubular, and in developing teeth the dentin in these regions changed to an irregularly formed hard tissue of osteodentin character, in which enclosed cells could be observed.

\section{Complications}

A major complication from natal/neonatal teeth is ulceration on the ventral surface of the tongue caused by the tooth's sharp incisal edge. This condition is also known as RigaFede disease or syndrome [47]. Possibility of swallowing and aspiration which has already been described previously should also be one of the major concerns in complications. Other complications stated are injury to mother's breast and inconvenience during suckling. The consequences seen with the teeth include carious lesions, pulp polyp, or premature eruption of successor teeth.

\section{Conclusion}

Natal and neonatal teeth diagnosis requires detailed case history accompanied by thorough clinical and radiographic examination of the infant. It is important to rule out by radiographic examination whether they are components of normal dentition or supernumerary to decide the treatment plan. The clinician should also assess the risk of haemorrhage due to the hypoprothrombinemia commonly present in newborns.

\section{Classification}

(i) The appearance of each natal tooth in the oral cavity can be classified into four categories given as follows, as the teeth emerge in the oral cavity: 
(1) shell-shaped crown poorly fixed to the alveolus by the gingival tissue and absence of a root;

(2) solid crown poorly fixed to the alveolus by the gingival tissue and little or no root;

(3) eruption of the incisal margin of the crown through the gingival tissues;

(4) edema of the gingival tissue with an unerupted but palpable tooth.

(ii) Spoug and Feasby have suggested that, clinically, natal and neonatal teeth are further classified according to their degree of maturity.

(1) A mature natal or neonatal tooth is the one which is nearly or fully developed and has relatively good prognosis for maintenance.

(2) The term immature natal or neonatal teeth, on the other hand, implies a tooth with incomplete or substandard structure; it also implies a poor prognosis.

(iii) If the degree of mobility is more than $2 \mathrm{~mm}$, the natal teeth of category (1) or (2) usually need extraction.

\section{Conflict of Interests}

The authors declared that there is no conflict of interests.

\section{References}

[1] J. Zhu and D. King, "Natal and neonatal teeth," ASDC Journal of Dentistry for Children, vol. 62, no. 2, pp. 123-128, 1995.

[2] E. Magitot, "Anomalies in the erupton of the teeth in man," The British Journal of Dental Science, vol. 26, pp. 640-641, 1883.

[3] J. W. Ballantyne, "Congenital teeth," Edinburgh Medical Journal, vol. 41, pp. 1025-1038, 1896.

[4] M. Massler and B. S. Savara, "Natal and neonatal teeth. A review of twenty-four cases reported in the literature," The Journal of Pediatrics, vol. 36, no. 3, pp. 349-359, 1950.

[5] W. C. Allwright, "Natal and neonatal teeth: a study among Chinese in Hong Kong," The British Dental Journal, vol. 105, pp. 163-173, 1958.

[6] J. Bodenhoff, "Natal and Neonatal teeth," European Journal of Oral Sciences, vol. 67, pp. 645-695, 1959.

[7] H. B. Wong, "Natal and neonatal teeth in Singapore," Journal of the Singapore Paediatric Society, vol. 4, pp. 74-82, 1962.

[8] J. Bodenhoff and R. J. Gorlin, "Natal and neonatal teeth, folklore and fact," Pediatrics, vol. 32, no. 6, pp. 1087-1093, 1963.

[9] J. T. Mayhall, "Natal and neonatal teeth among the Tlinget Indians," Journal of Dental Research, vol. 46, no. 4, pp. 748-749, 1967.

[10] M. H. Chow, "Natal and neonatal teeth," Journal of the American Dental Association, vol. 100, pp. 215-216, 1980.

[11] R. A. Anderson, "Natal and neonatal teeth: histological investigation of two black females," Journal of Dentistry for Children, vol. 49, pp. 300-303, 1982.

[12] G. A. Kates, H. L. Needleman, and L. B. Holmes, "Natal and neonatal teeth: a clinical study," The Journal of the American Dental Association, vol. 109, no. 3, pp. 441-443, 1984.
[13] A. K. Leung, "Natal teeth," The American Journal of Diseases of Children, vol. 140, pp. 249-251, 1986.

[14] R. Bedi and W. Yan, "The prevalence and clinical management of natal teeth-a study in Hong Kong," Journal of Pediatrics, vol. 6, pp. 85-90, 1990.

[15] M. Rusmah, "Natal and neonatal teeth: a clinical and histological study," The Journal of Clinical Pediatric Dentistry, vol. 15, no. 4, pp. 251-253, 1991.

[16] E. W. To, "A study of natal teeth in Hong Kong Chinese," International Journal of Paediatric Dentistry, vol. 1, no. 2, pp. 7376, 1991.

[17] C. M. De Almeida and M. R. Gomide, "Prevalence of natal/neonatal teeth in cleft lip and palate infants," Cleft PalateCraniofacial Journal, vol. 33, no. 4, pp. 297-299, 1996.

[18] S. Alaluusua, H. Kiviranta, A. Leppäniemi et al., "Natal and neonatal teeth in relation to environmental toxicants," Pediatric Research, vol. 52, no. 5, pp. 652-655, 2002.

[19] K. El Khatib, A. Abouchadi, M. Nassih et al., "Natal teeth: apropos of five cases," Revue de Stomatologie et de Chirurgie Maxillo-Faciale, vol. 106, no. 6, pp. 325-327, 2005.

[20] H. C. Ruschel, M. H. Spiguel, D. D. Piccinini, S. H. Ferreira, and E. G. Feldens, "Natal primary molar: clinical and histological aspects," Journal of Oral Science, vol. 52, no. 2, pp. 313-317, 2010.

[21] S. B. Deep, E. Ranadheer, and B. Rohan, "Riga-Fede disease: report of a case with literature review," Journal of Academy of Advanced Dental Research, vol. 2, no. 2, pp. 27-30, 2011.

[22] S. Nandikonda, "Natal teeth with cleft palate: a case report," International Journal of Contemporary Dentistry, vol. 1, no. 3, pp. 124-126, 2010.

[23] H. Dyment, R. Anderson, J. Humphrey, and I. Chase, "Residual neonatal teeth: a case report," Journal of the Canadian Dental Association, vol. 71, no. 6, pp. 394-397, 2005.

[24] U. D. Shrestha, "Postoperative follow up challenge in paediatric cataract surgery in Nepal," Journal of Nepal Paediatric Society, vol. 31, no. 3, pp. 198-201, 2011.

[25] S. Chandra, "Natal teeth and neonatal teeth: a report of three cases," Journal of the Indian Dental Association, vol. 5, no. 1, 2011.

[26] V. Gina, G. Julieta, M. Valentina, R. Helen, and M. Windy, "Neonatal teeth: a case report and literature review," Revista Venezolana de Investigación Odontológica, vol. 8, no. 2, pp. 2936, 2008 (Spanish).

[27] K. Marakoglu, E. F. Percin, I. Marakoglu, U. K. Gursoy, and F. Goze, "Anencephalic infant with cleft palate and natal teeth: a case report," Cleft Palate-Craniofacial Journal, vol. 41, no. 4, pp. 456-458, 2004.

[28] P. Kaur, A. Sharma, and N. Bhuller, "Conservative management of a complication of neonatal teeth: a case report," Journal of the Indian Society of Pedodontics and Preventive Dentistry, vol. 21, no. 1, pp. 27-29, 2003.

[29] E. Ndiokwelu, G. N. Adimora, and N. Ibeziako, "Neonatal teeth association with Down's syndrome. A case report," OdontoStomatologie Tropicale, vol. 27, no. 107, pp. 4-6, 2004.

[30] C. A. María and Bibiana, "Tooth neonatal patient with systemic involvement (hydrocephalus, meningitis): a case report," Revista Estomatologia, vol. 11, no. 1, pp. 55-59, 2003.

[31] S. P. Rdos, R. A. Otero, M. B. Portela, and G. F. Castro, "Severe oligodontia and dental anomalies in a child with a history of multiple natal teeth: an eight-year retrospective," General Dentistry, vol. 59, no. 6, pp. e248-e250, 2011.

[32] M. Agostini, J. E. León, M. G. Kellermann, R. Valiati, E. Graner, and O. P. de Almeida, "Myxoid calcified hamartoma 
and natal teeth: a case report," International Journal of Pediatric Otorhinolaryngology, vol. 72, no. 12, pp. 1879-1883, 2008.

[33] A. Tomaki, O. Hiroyuki, E. Kitamura et al., "A case of a natal tooth showing pedunculated poly-like appearance," International Journal of Oral Medical Sciences, vol. 4, no. 2, pp. 107-110, 2005.

[34] J. Kovac and D. Kovac, "Neonatal teeth," Bratislava Medical Journal, vol. 112, no. 11, pp. 648-650, 2011.

[35] J. R. Sibert and J. R. Porteous, "Erupted teeth in the newborn: 6 members in a family," Archives of Disease in Childhood, vol. 49, no. 6, pp. 492-493, 1974.

[36] L. G. Primo, A. C. Alves, I. Pomarico, and R. Gleiser, "Interruption of breast feeding caused by the presence of neonatal teeth," Brazilian Dental Journal, vol. 6, no. 2, pp. 137-142, 1995.

[37] N. N. Basavanthappa, U. Kagathur, R. N. Basavanthappa, and S. T. Suryaprakash, "Natal and neonatal teeth: a retrospective study of 15 cases," European Journal of Dentistry, vol. 5, no. 2, pp. 168-172, 2011.

[38] R. D. McDonald, D. R. Avery, and J. A. Dean, Dentistry for the Child and Adolescent, Mosby, St. Louis, Mo, USA, 8th edition, 2004.

[39] G. W. Friend, H. H. Mincer, K. R. Carruth, and J. E. Jones, "Natal primary molar: case report," Pediatric Dentistry, vol. 13, no. 3, pp. 173-175, 1991.

[40] K. Kurian, S. Shanmugam, T. H. Vardah, and S. Gupta, "Chondroectodermal dysplasia (Ellis van Creveld syndrome): a report of three cases with review of literature," Indian Journal of Dental Research, vol. 18, no. 1, pp. 31-34, 2007.

[41] A. Taghi and M. H. Motamedi, "Riga-Fede disease: a histological study and case report," Indian Journal of Dental Research, vol. 20, no. 2, pp. 227-229, 2009.

[42] S. Sogi, S. M. Hugar, S. Patil, and S. Kumar, "Multiple natal teeth: a rare case report," Indian Journal of Dental Research, vol. 22, no. 1, pp. 169-171, 2011.

[43] C. Venkatesh and B. Adhisivam, "Natal teeth in an infant with congenital hypothyroidism," Indian Journal of Dental Research, vol. 22, no. 3, p. 498, 2011.

[44] A. S. Roshan, C. Janaki, B. Parveen, and N. Gomathy, "Rare association of hyper IgE syndrome with cervical rib and natal teeth," Indian Journal of Dermatology, vol. 54, no. 4, pp. 372$374,2009$.

[45] K. M. Veena, H. Jagadishchandra, P. K. Rao, and L. Chatra, "Ellis-van Creveld syndrome in an Indian child: a case report," Imaging Sciences in Dentistry, vol. 41, no. 4, pp. 1167-1170, 2011.

[46] B. B. Rao, G. R. Mamatha, K. N. Zameera, and R. B. Hegde, "Natal and neonatal teeth: a case report," Journal of the Indian Society of Pedodontics and Preventive Dentistry, vol. 19, no. 3, pp. 110-112, 2001.

[47] R. T. Anegundi, R. Sudha, H. Kaveri, and K. Sadanand, "Natal and neonatal teeth: a report of four cases," Journal of the Indian Society of Pedodontics and Preventive Dentistry, vol. 20, no. 3, pp. 86-92, 2002.

[48] S. Singh, V. V. S. Reddy, G. Dhananjaya, and R. Patil, "Reactive fibrous hyperplasia associated with a natal tooth," Journal of Indian Society of Pedodontics and Preventive Dentistry, vol. 22, no. 4, pp. 183-186, 2004.

[49] M. N. Ziai, D. J. Bock, A. da Silveira, and J. L. Daw, "Natal teeth: a potential impediment to nasoalveolar molding in infants with cleft lip and palate," Journal of Craniofacial Surgery, vol. 16, no. 2, pp. 262-266, 2005.
[50] R. J. Hegde, "Sublingual traumatic ulceration due to neonatal teeth (Riga-Fede disease)," Journal of Indian Society of Pedodontics and Preventive Dentistry, vol. 23, no. 1, pp. 51-52, 2005.

[51] S. Sarkar and S. Sarkar, "Unusual neonatal tooth in maxillary 1st molar region: a case report," Journal of Indian Society of Pedodontics and Preventive Dentistry, vol. 25, supplement, pp. S41-S42, 2007.

[52] A. Kumar, H. Grewal, and M. Verma, "Posterior neonatal teeth," Journal of Indian Society of Pedodontics and Preventive Dentistry, vol. 29, no. 1, pp. 68-70, 2011.

[53] R. S. Rao and S. V. Mathad, "Natal teeth: case report and review of literature," Journal of Oral and Maxillofacial Pathology, vol. 13, no. 1, pp. 41-46, 2009.

[54] M. Muraleekrishnan, P. T. S. Babu, T. C. Pratap, and G. Parvathy, "Congenital eruption cyst associated with natal teeth," Kerala Dental Journal, vol. 34, no. 1, pp. 23-25, 2011.

[55] Y. Masatomi, K. Abe, and T. Ooshima, "Unusual multiple natal teeth: case report," Pediatric Dentistry, vol. 13, no. 3, pp. 170-172, 1991.

[56] F. A. Gonçalves, E. G. Birman, N. N. Sugaya, and A. M. Melo, "Natal teeth: review of the literature and report of an unusual case," Brazilian Dental Journal, vol. 9, no. 1, pp. 53-56, 1998.

[57] A. R. Prabhakar, G. R. Ravi, O. S. Raju, A. J. Kurthukoti, and A. B. Shubha, "Neonatal tooth in fraternal twins: a case report," International Journal of Clinical Pediatric Dentistry, vol. 2, no. 2, pp. 40-44, 2009.

[58] M. Agostini, J. E. León, M. G. Kellermann, R. Valiati, E. Graner, and O. P. de Almeida, "Myxoid calcified hamartoma and natal teeth: a case report," International Journal of Pediatric Otorhinolaryngology, vol. 72, no. 12, pp. 1879-1883, 2008.

[59] L. Dubois, K. H. Keuning, and J. A. Lindeboom, "Traumatic ulceration of the tongue in an infant," Nederlands Tijdschrift voor Tandheelkunde, vol. 117, no. 5, pp. 274-275, 2010.

[60] K. A. Eley, P. A. Watt-Smith, and S. R. Watt-Smith, "Deformity of the tongue in an infant: Riga-Fede disease," Paediatrics and Child Health, vol. 15, no. 9, pp. 581-582, 2010.

[61] F. Samadi, P. Babaji, S. Saha, A. Katiyar, and S. Chowdhury, "Natal teeth: report of two cases and review of literature," International Journal of Oral and Maxillofacial Pathology, vol. 2, no. 1, pp. 33-36, 2011.

[62] R. L. Slayton, "Treatment alternatives for sublingual traumatic ulceration (Riga-Fede disease)," Pediatric Dentistry, vol. 22, no. 5, pp. 413-414, 2000.

[63] M. Y. Padmanabhan, R. K. Pandey, R. Aparna, and V. Radhakrishnan, "Neonatal sublingual traumatic ulceration-case report \& review of the literature," Dental Traumatology, vol. 26, no. 6, pp. 490-495, 2010.

[64] S. K. Brandt, S. D. Shapiro, and P. E. Kittle, "Immature primary molar in the newborn," Pediatric Dentistry, vol. 5, no. 3, pp. 210213, 1983.

[65] M. P. Alvarez, P. V. Crespi, and A. L. Shanske, "Natal molars in Pfeiffer syndrome type 3: a case report," The Journal of Clinical Pediatric Dentistry, vol. 18, no. 1, pp. 21-24, 1993.

[66] J. D. Spouge and W. H. Feasby, "Erupted teeth in the newborn," Oral Surgery, Oral Medicine, Oral Pathology, vol. 22, no. 2, pp. 198-208, 1966.

[67] J. Hebling, A. C. C. Zuanon, and D. R. Vianna, "Dente Natal-a case of natal teeth," Odontologia Clinica, vol. 7, pp. 37-40, 1997.

[68] A. K. C. Leung and W. L. M. Robson, "Natal teeth: a review," Journal of the National Medical Association, vol. 98, no. 2, pp. 226-228, 2006. 
[69] R. Sureshkumar and A. H. McAulay, "Natal and neonatal teeth," Archives of Disease in Childhood: Fetal and Neonatal Edition, vol. 87, no. 3, article F227, 2002.

[70] R. F. Cunha, F. A. C. Boer, D. D. Torriani, and W. T. G. Frossard, "Natal and neonatal teeth: review of the literature," Pediatric Dentistry, vol. 23, no. 2, pp. 158-162, 2001.

[71] L. G. Primo, A. C. Alves, I. Pomarico, and R. Gleiser, "Interruption of breast feeding caused by the presence of neonatal teeth," Brazilian Dental Journal, vol. 6, no. 2, pp. 137-142, 1995.

[72] N. M. King and A. M. P. Lee, "Prematurely erupted teeth in newborn infants," Journal of Pediatrics, vol. 114, no. 5, pp. 807809, 1989.

[73] M. Baumgart and A. Lussi, "Natal and neonatal teeth," Schweizer Monatsschrift für Zahnmedizin, vol. 116, no. 9, pp. 894-909, 2006 (German).

[74] I. Štamfelj, J. Jan, E. Cvetko, and D. Gašperšič, "Size, ultrastructure, and microhardness of natal teeth with agenesis of permanent successors," Annals of Anatomy, vol. 192, no. 4, pp. 220-226, 2010.

[75] H. Weiss, "Chondroectodermal dysplasia: report of a case and review of the literature," The Journal of Pediatrics, vol. 46, no. 3, pp. 268-275, 1955.

[76] P. Robotta and E. Schafer, "Hallermann-Streiff syndrome: case report and literature review," Quintessence International, vol. 42, no. 4, pp. 331-338, 2011.

[77] E. Ndiokwelu, G. N. Adimora, and N. Ibeziako, "Neonatal teeth association with Down's syndrome. A case report," OdontoStomatologie Tropicale, vol. 27, no. 107, pp. 4-6, 2004.

[78] C. Venkatesh and B. Adhisivam, "Natal teeth in an infant with congenital hypothyroidism," Indian Journal of Dental Research, vol. 22, no. 3, p. 498, 2011.

[79] F. A. Gonçalves, "Natal teeth: review of the literature and report of an unusual case," Brazilian Dental Journal, vol. 9, no. 1, pp. 53-55, 1998.

[80] E. Hals, "Natal and neonatal teeth. Histologic investigations in two brothers," Oral Surgery, Oral Medicine, Oral Pathology, vol. 10, no. 5, pp. 509-521, 1957.

[81] J. R. Jasmin and S. Clergeau-Guerithault, "A scanning electron microscopic study of the enamel of neonatal teeth," Journal de Biologie Buccale, vol. 19, no. 4, pp. 309-314, 1991.

[82] M. Uzamis, S. Olmez, H. Ozturk, and H. Celik, "Clinical and ultrastructural study of natal and neonatal teeth," Journal of Clinical Pediatric Dentistry, vol. 23, no. 3, pp. 173-177, 1999.

[83] G. W. Friend, H. H. Mincer, K. R. Carruth, and J. E. Jones, "Natal primary molar: case report," Pediatric Dentistry, vol. 13, no. 3, pp. 173-175, 1991. 


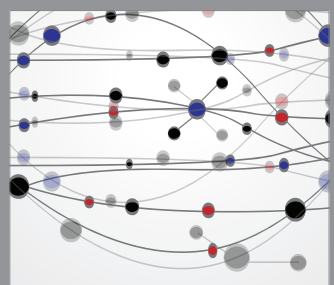

The Scientific World Journal
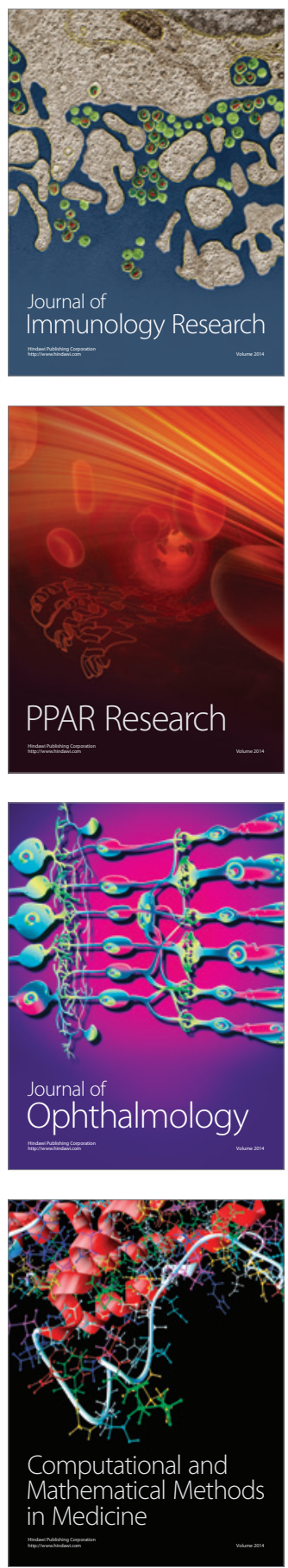

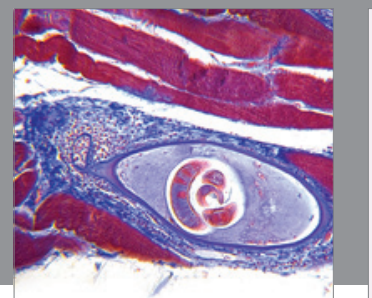

Gastroenterology

Research and Practice
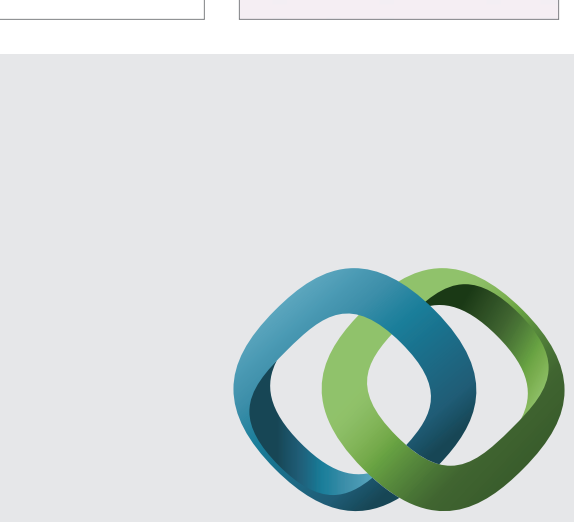

\section{Hindawi}

Submit your manuscripts at

http://www.hindawi.com
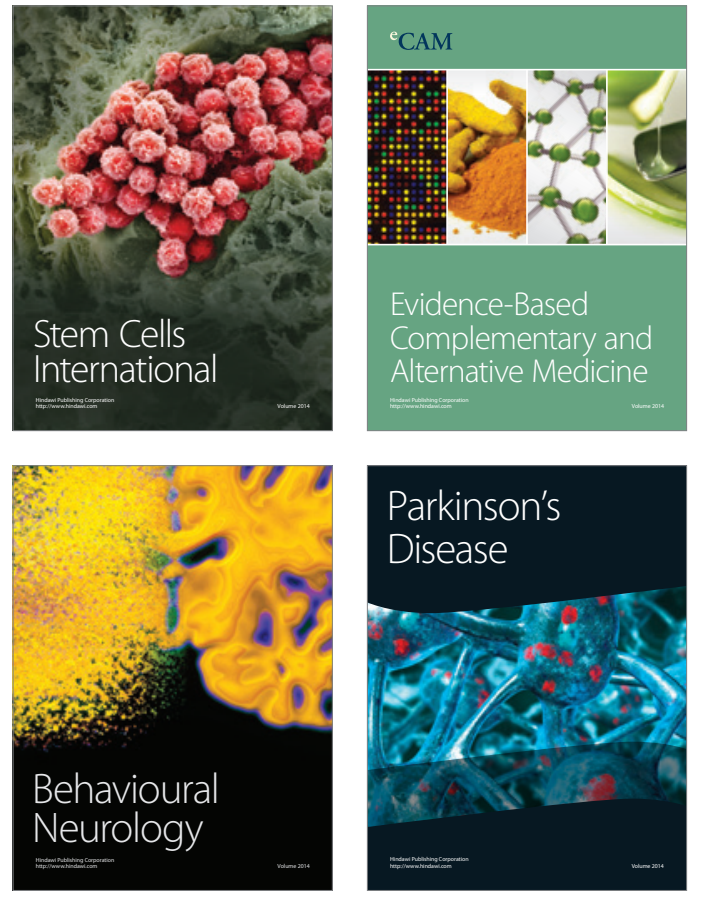
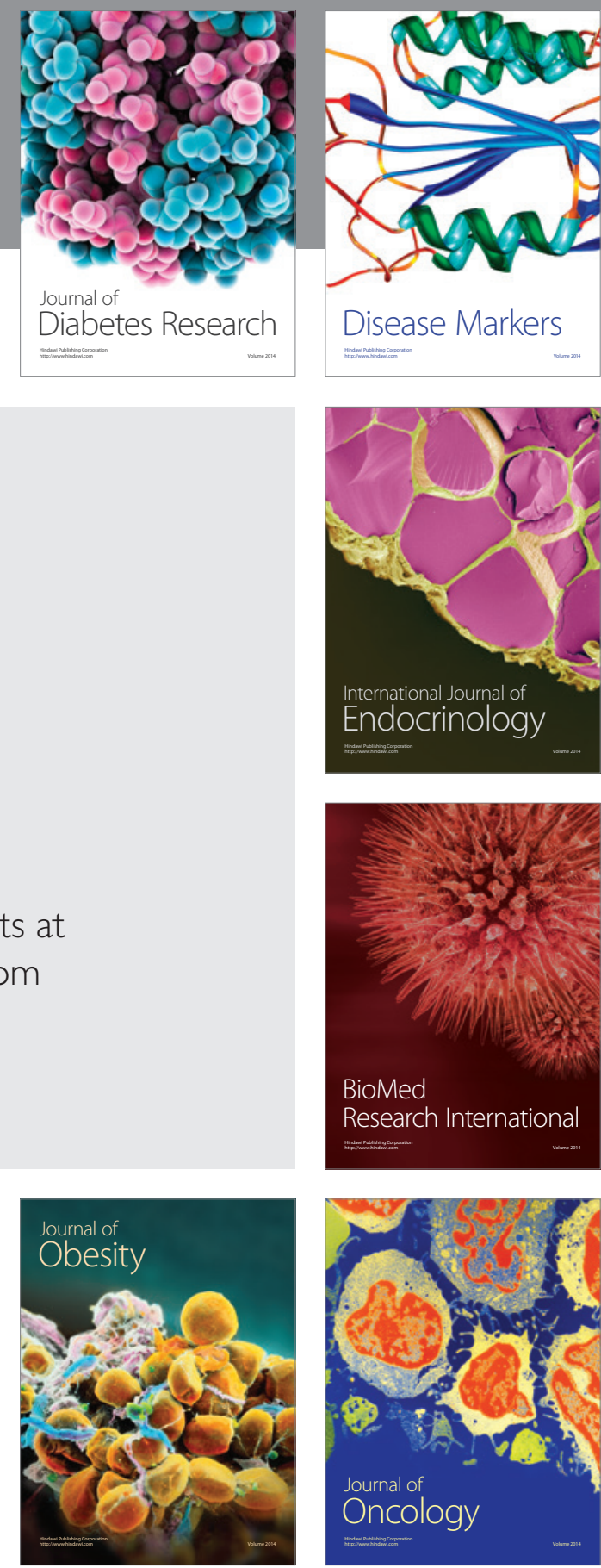

Disease Markers
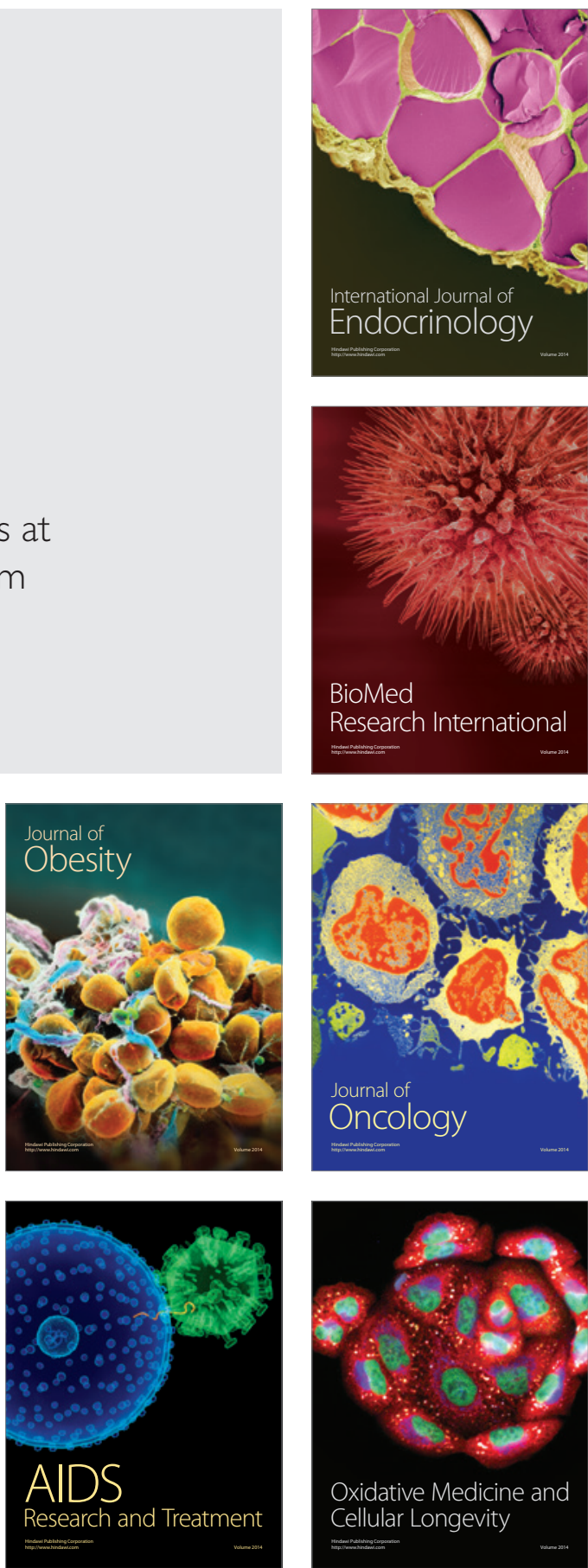